\title{
Potential regrowth of dental tissue with stem cells from baby teeth
}

Researchers may have found a way to use stem cells extracted from a patient's baby teeth to regrow dental tissue.

Around half of children experience an injury to a tooth during childhood and when that trauma affects an immature permanent tooth it can hinder blood supply and root development, resulting in what is, effectively, a 'dead' tooth.

Currently, clinicians can perform a procedure called apexification to encourage further root development, but it does not replace the lost tissue from the injury and can cause root development to proceed abnormally.

Results of a clinical trial, published on 22 August 2018 in the journal Science Translational Medicine, ${ }^{1}$ suggested there was a more promising path for children with these types of injuries, namely, using stem cells extracted from the patient's baby teeth.

The study was led jointly by Songtao Shi of the University of Pennsylvania in the USA and Yan Jin, Kun Xuan, and Bei Li of the Fourth Military Medicine University in Xian in China.

The trial was conducted in China involving 40 children who had each injured one of their permanent incisors, and still had baby teeth. Thirty were assigned to the human deciduous pulp stem cell (hDPSC) implantation treatment and ten to the control group who had traditional treatment of apexification.

The children who received hDPSC treatment had tissue extracted from a healthy baby tooth. The stem cells from this pulp were allowed to reproduce in a laboratory culture, and the resulting cells were implanted into the injured tooth.

Upon follow-up, the researchers found that patients who received hDPSCs had more signs than the control group of healthy root development and thicker dentine, as well as increased blood flow.

At the time the patients were initially seen, all had little sensation in the tissue of their injured teeth, but a year after the procedure only those who received hDPSCs had regained some sensation.

Examining a variety of immune-system components, the team found no evidence of safety concerns.

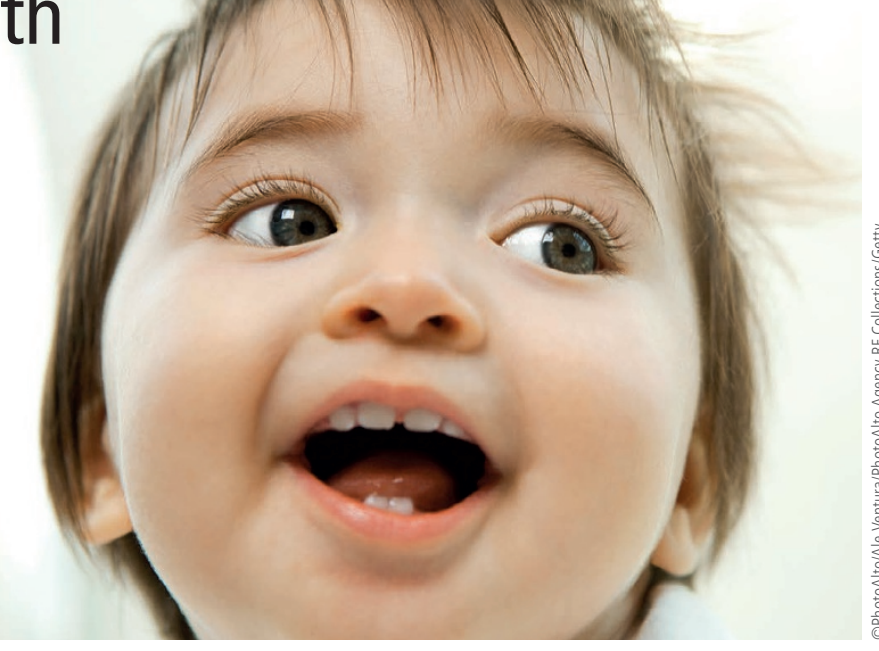

The researchers also had the opportunity to directly examine the tissue of a treated tooth when the patient re-injured it, and had to have it extracted.

They found that the implanted stem cells regenerated different components of dental pulp, including the cells that produce dentine, connective tissue, and blood vessels.

Shi, Professor and Chair in the Department of Anatomy and Cell Biology at University of Pennsylvania's School of Dental Medicine, said: 'This treatment gives patients sensation back in their teeth. They have living teeth again.

'So far we have follow-up data for two, two and a half, even three years, and have shown it's a safe and effective therapy. For me, the results are very exciting. To see something we discovered take a step forward to potentially become a routine therapy in the clinic is gratifying.'

It was, however, a first step and the authors said that while using a patient's own stem cells reduced the chance of immune rejection, it was not possible in adult patients who had lost all of their baby teeth.

1. Xuan $K$, Li B, Songtao S, Jin Y et al. Deciduous autologous tooth stem cells regenerate dental pulp after implantation into injured teeth. Sci Trans/ Med 2018; 10: eaaf3227. DOI: 10.1126/scitransImed.aaf3227. Available at http://stm.sciencemag.org/content/10/455/eaaf3227 (accessed 19 September 2018).

\section{Union seeks views of academic dentists on working in higher education}

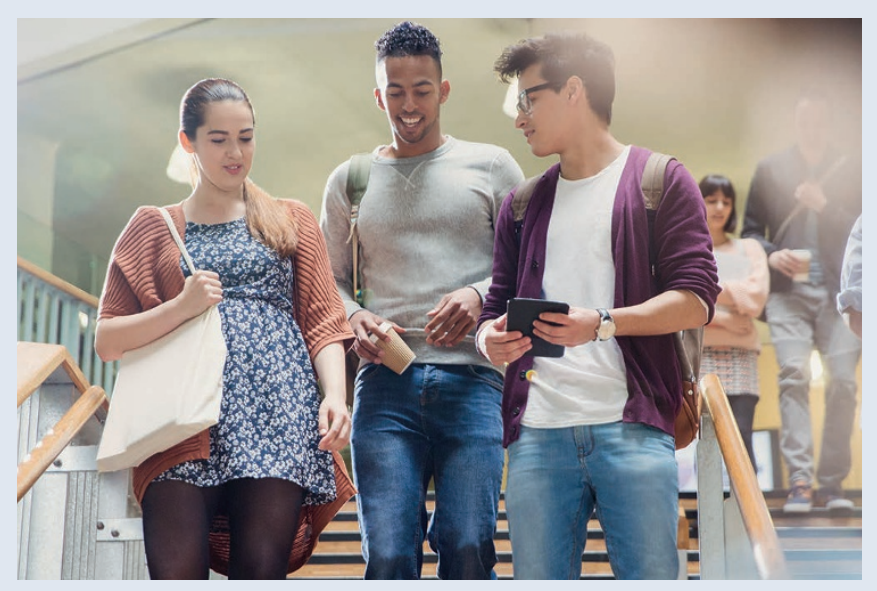

Dentists who work within the higher education sector across the UK are being asked to complete a survey by the BDA to help gain a greater understanding of the issues facing academic dentists.

BDA members who work in academia in England, Northern Ireland, Scotland or Wales, are being encouraged to share their views via the survey and BDA members have been sent a link via email that should be completed by 19 October 2018 .

Results from the survey are designed to help the union build a better picture of the issues facing dentists working in academia, including working conditions and the impact of ongoing changes taking place within the higher education sector.

Any BDA members who have not yet received the link to the survey should email ian.oakley@bda.org. 